\title{
Diagnostic Accuracy of Dual-Source Computerized Tomography Coronary Angiography in Symptomatic Patients Presenting to a Referral Cardiovascular Center During Daily Clinical Practice
}

\author{
Arash Mahdavi, ${ }^{1}$ Ali Mohammadzadeh, ${ }^{2}$ Golsa Joodi, ${ }^{3}$ Mohammad Reza Tabatabaei, ${ }^{3}$ Farhad \\ Sheikholeslami, ${ }^{4}$ and Marzieh Motevalli ${ }^{2, *}$ \\ ${ }^{1}$ Faculty of Medicine, Shahid Beheshti University of Medical Sciences, Tehran, Iran \\ ${ }^{2}$ Rajaie Cardiovascular Medical and Research Center, Iran University of Medical Sciences, Tehran, Iran \\ ${ }^{3}$ Faculty of Medicine, Tehran University of Medical Sciences, Tehran, Iran \\ ${ }^{4}$ Endocrine Research Center, Research Institute for Endocrine Sciences, Shahid Beheshti University of Medical Sciences, Tehran, Iran \\ "Corresponding author: Marzieh Motevalli, Rajaie Cardiovascular Medical and Research Center, Iran University of Medical Sciences, Tehran, Iran. Tel: +98-2122042026, Fax: \\ +98-21-22042026, E-mail: motevalli_m@yahoo.com
}

Received 2014 October 05; Revised 2014 October 21; Accepted 2014 November 11.

\begin{abstract}
Background: There are numerous studies that address the diagnostic value of dual-source computed tomography (DSCT) as an alternative to conventional coronary angiography (CCA). However, the benefit of application of DSCT in a real world clinical setting should be evaluated.

Objectives: To determine the diagnostic accuracy of DSCT technique compared with CCA as the gold standard method in detection of coronary artery stenosis among symptomatic patients who are presented to a referral cardiovascular center during daily clinical practice.

Patients and Methods: Evaluating the medical records of a tertiary care referral cardiovascular center, 47 patients who had undergone DSCT and CCA, and also met the inclusion and exclusion criteria of the study were selected. Sensitivity, specificity, positive predictive value (PPV), negative predictive value (NPV), and likelihood ratios (LRs) of the DSCT imaging technique were calculated. Results: In total, $97.8 \%$ of the segments (628/642) could be visualized with diagnostic image quality via DSCT coronary angiography. The mean heart rate during DSCT was $69.2 \pm 12.2 \mathrm{bpm}$ (range: 39 - $83 \mathrm{bpm}$ ), and the mean Agatston score was $507.7 \pm 590.5$ (range: 0 -2328). Per segment analysis of the findings revealed that the sensitivity, specificity, PPV, NPV, positive LR (PLR) and negative LR(NLR) of DSCT technique for evaluation of patients with coronary artery disease were 93.7\%, 96.8\%, 92.7\%, 97.2\%, 29.4, and 0.066, respectively. Also per vessel, analysis of the findings showed a sensitivity of $97.1 \%$, a specificity of $94.0 \%$, PPV of 95.3\%, NPV of $96.3 \%$, PLR of 16.1 , and NLR of 0.030 .

Conclusion: Our results indicate that DSCT coronary angiography provides high diagnostic accuracy for the evaluation of CAD patients during daily routine practice of a referral cardiovascular setting.
\end{abstract}

Keywords: Multidetector Computed Tomography, Coronary Angiography, Cardiac Imaging Techniques, Atherosclerosis

\section{Background}

Ischemic heart disease remains one of the leading causes of morbidity and mortality in western as well as developing countries $(1,2)$. Early diagnosis of arterial stenosis using available imaging techniques has been the issue of many previous studies. According to these studies, conventional coronary angiography (CCA) has shown excellent results in demonstrating stenosis in coronary arteries and is known as the gold standard method for the diagnosis of vascular luminal stenosis (3). CCA is an invasive method, which is associated with a wide list of possible complications in the subjects (4). Besides, according to previous studies, more than 50 percent of the subjects who undergo elective CCA have no significant coronary artery stenosis
(5). Therefore, a comparably accurate non-invasive method for detecting coronary artery disease (CAD) is of major interest.

In recent years, substantial advances have been achieved in providing non-invasive imaging modalities for CAD evaluation such as multi-detector CT (MDCT) and magnetic resonance angiography (MRA). Schuetz et al. (6) compared MDCT and MRA and showed a higher accuracy for MDCT in the detection of coronary artery stenosis. Although single-source CT was initially proved to be a beneficial non-invasive technique in detecting coronary stenoses, its limited temporal resolution might restrict its clinical use (7). To overcome this limitation, administration of beta-blocker medications was proposed to decrease the heart rate of the subjects and thereby 
limit the motion artifact of the imaging $(8,9)$. Technical advances over the past years led to the introduction of new generation of CT machines such as dual-source CT (DSCT) scanners that resulted in imaging with improved temporal resolution (up to $75 \mathrm{~ms}$ in mono-segment reconstruction mode). Improved temporal resolution of DSCT makes them independent of heart rate and therefore, the subjects are not needed to receive beta-blockers before the imaging study (10). This could be considered as one of the advantages of this technique particularly for patients in whom administration of beta blockers is contraindicated.

The sensitivity and specificity of single-source CT (with 64 slices) and dual-source CT for diagnosis of coronary artery diseases has been well studied before. The most notable finding was the high negative predictive value of these methods that allows reliable exclusion of significant stenosis of coronary arteries as well as prevention of CCA in people with negative CT scans (11-15). These studies are mostly done on patients who were recruited for participation in a research study. To our knowledge, there are scarce data on the accuracy of DSCT technique for detection of coronary artery diseases in a clinical setting and among patients presenting to a tertiary care referral heart center (16).

\section{Objectives}

The aim of the current study was to determine the sensitivity and specificity of second-generation DSCT compared with CCA as the gold standard method in the detection of coronary artery stenosis among symptomatic patients who were referred to a referral cardiovascular center during daily clinical practice.

\section{Patients and Methods}

All subjects received written and oral information about the study procedures. The study protocol was approved by the ethical committee of our university.

\subsection{Study Population}

This retrospective study was performed in an academic and tertiary care referral cardiovascular center.

Evaluating the medical records of our center, 518 patients were found who were referred to our clinic with symptoms of typical or atypical chest pain (that were considered as suggestive symptoms of CAD or chronic unstable angina) over an 18-month period and underwent DSCT. These patients did not have any contraindication for DSCT (renal insufficiency, known allergy to iodinated contrast material or pregnancy). Of those, patients with irregular elevated heart rates, history of stenting and congenital coronary anomalies were excluded from the study and data on 390 remained subjects were further evaluated. Among these patients, 47 subjects ( 35 males and 12 females) who underwent CCA over a period of 60 days from the DSCT study were included for final analysis.

\subsection{Dual-Source Computerized Tomography Scan Protocol and Image Reconstruction}

DSCT imaging was performed using a secondgeneration dual-source CT system (Somatom definition flash, Siemens healthcare, Forchheim, Germany). DSCT scanners have two X-ray tubes and two corresponding detectors mounted onto the rotating gantry with an angular offset of $90^{\circ}$ (17). This specific structure makes DSCT systems almost two times faster than conventional single-source CT systems in reconstruction of each image slab (7). To calculate calcium score, non-enhanced DSCT was performed before CT coronary angiography from $1 \mathrm{~cm}$ below the level of the tracheal bifurcation to the diaphragm. For calcium scoring, the following scanning parameters were used: detector collimation, $64 \times 0.6$ $\mathrm{mm}$; slice acquisition, $256 \times 0.6 \mathrm{~mm}$ by means of a $\mathrm{z}$-flying focal spot; gantry rotation time, $280 \mathrm{~ms}$; pitch of 0.2 0.35 adapted to the heart rate; tube current, $100 \mathrm{mAs}$ per rotation; and tube potential, $120 \mathrm{kV}$. None of the subjects were excluded from the study, regardless of the patient's calcium score. Afterwards, all patients received a single puff of nitroglycerin (0.4 mg/dose, Glytrin Bioglan Laboratories Ltd, Hitchin, United Kingdom). Patients who used daily beta-blockers as part of their treatment plan, were not forced to omit their beta-blockers before the exam. No additional beta-blockers were administered prior to the scan, irrespective of the individual's heart rate. After 2 minutes, a bolus of $80 \mathrm{~mL}$ of iohexol (omnipaque 350, 350 $\mathrm{mgI} / \mathrm{mL}$, GE Healthcare, Ireland cork, Ireland) followed by $30 \mathrm{~mL}$ saline chaser, was injected into an antecubital vein via a 19-gauge catheter (injection rate $6 \mathrm{~mL} / \mathrm{s}$ ) (18). Contrast-agent application was controlled by bolus tracking in the aortic root. Image acquisition started 5 seconds after reaching the signal attenuation to the predefined threshold of 160 Hounsfield units (HU). Data acquisition was performed from $1 \mathrm{~cm}$ below the level of the tracheal bifurcation to the diaphragm in a cranio-caudal direction with a detector collimation of $64 \times 0.6 \mathrm{~mm}$, slice acquisition of $256 \times 0.6 \mathrm{~mm}$ by means of a z-flying focal spot, gantry rotation time of $280 \mathrm{~ms}$, pitch of $0.2-0.35$ adapted to the heart rate, tube current of $400 \mathrm{mAs}$ per rotation, and tube potential of $120 \mathrm{kV}$. Full tube current was applied between 30 and $85 \%$ of the cardiac cycle. A gantry rotation time of $0.28 \mathrm{~s}$ leads to a temporal resolution of $75 \mathrm{~ms}$. ECG-pulsing for radiation dose reduction was applied in all patients. 
For CT coronary angiography(CTCA), axial images were reconstructed with $0.6 \mathrm{~mm}$ slice thickness and $0.3 \mathrm{~mm}$ increment using a medium soft convolution kernel and retrospective ECG gating. The best systolic and diastolic data set were chosen for final image interpretation. A monosegment reconstruction algorithm using data from a quarter rotation of both detectors was used for image reconstruction (17). All reconstructed images were transferred to a workstation (Leonardo, Siemens medical solutions) equipped with cardiac post-processing software (Syngo circulation, Siemens medical solutions).

\subsection{Dual-source Computerized Tomography Data Analysis}

The mean Agatston score was calculated for each patient from the non-enhanced DSCT by use of semiautomated software (Syngo calcium scoring, Siemens medical solutions). Agatston et al. (19) defined calcification as a computed tomographic density of $130 \mathrm{HU}$ having an area equal or larger than $1 \mathrm{~mm}^{2}$. A score of 1 is given for 130 to $200 \mathrm{HU}, 2$ for 201 to $299 \mathrm{HU}, 3$ for 300 to $399 \mathrm{HU}$, and 4 for $400 \mathrm{HU}$ or greater.

Interpretation of CTCA images of each patient was made by consensus of one expert cardiac radiologist and a cardiologist specialized in cardiac imaging using axial source images, thin-slab maximum intensity projections (MIP), and multi-planar reformations (MPR). Coronary segments were identified using American heart association guidelines (20). The right coronary artery (RCA) included segments 1 - 4, left main coronary artery (LMCA) was defined as segment 5 , left anterior descending artery (LAD) included segments $6-10$, and the left circumflex artery (LCX) consisted of segments $11-15$. The ramus intermedius was defined as segment 16 , if present. All coronary artery segments, including RCA branches and posterior descending artery (PDA) branch of LCX in left or co-dominant patients that were $1.5 \mathrm{~mm}$ or larger in diameter at visual inspection, were added to the analysis.

First, we decided whether segments were assessable or non-assessable. As routine practice of our hospital, stenoses were visually evaluated and described as no stenosis (0\%), mild stenosis ( $0 \%-49 \%)$, moderate stenosis (50\% - 69\%), severe stenosis (70\% - 99\%) and total occlusion. A third observer, who was unaware of CCA findings, classified each segment as significant $(\geq 50 \%$ luminal diameter reduction) or non-significant $(<50 \%$ luminal diameter reduction) based on the radiologic data and report. Segments distal to an occlusion were excluded from analysis. In case of multiple lesions in a segment, we classified the segment according to the worst stenosis present.

\subsection{Conventional Coronary Angiography}

All included patients had also undergone CCA by a cardiac interventionist. Angiography data were stored on a CD-ROM in multiple views and subsequently analyzed by a cardiologist who was blinded to CTCA results. Coronary segments were identified by the same guideline used in DSCT coronary angiography (20) and all vessels with a luminal diameter greater or equal to $1.5 \mathrm{~mm}$ were included in the analysis. Similar to DSCT coronary angiography, significant stenosis was defined as luminal diameter reduction of more than $50 \%$. All assessments were performed visually and segments distal to an occlusion were excluded from analysis. In the case of discordance between DSCT and CCA in defining the coronary artery segment, an agreement was reached by consensus (21).

\subsection{Statistical Analysis}

Statistical analysis was performed using statistical software (SPSS ver. 16.0, SPSS Inc., Chicago, IL). Considering CCA as the gold standard method of imaging, the sensitivity, specificity, positive predictive value (PPV), negative predictive value (NPV), positive likelihood ratio (PLR), and negative likelihood ratio (NLR) of DSCT technique were calculated with 95\% confidence interval (CI). Quantitative variables were expressed as mean \pm standard deviation (SD) and discrete variables were presented as counts and percentages. Analyses were performed per segment, per vessel and in segments pertaining to various coronary vessel territories.

\section{Results}

We reviewed data from 47 patients (mean age, $60.6 \pm$ 11.5 years; age range, 39 - 83 years) who underwent both DSCT coronary angiography and CCA (mean interval. 16.7 \pm 17.3 ; range, 1 - 57 days). No complication was reported during DSCT coronary angiography or CCA examinations in the selected patients. The baseline characteristics of the subjects are shown in Table 1.

During DSCT coronary angiography, average heart rate (HR) of the subjects was $69.2 \pm 12.2$ beats per minute (bpm) (range: 48-95). Twenty-three patients (49\%) had HR below $70 \mathrm{bpm}$, and 24 patients (51\%) had HR above $70 \mathrm{bpm}$ at the time of performing DSCT study. Calcium score of three patients was not available in our records and the mean Agatston score of the remaining 44 subjects was $507.7 \pm 590.5$, with $57 \%$ of the patients with a score below 400 . 
Table 1. Patients' Characteristics $(n=47)$

\begin{tabular}{|c|c|}
\hline Gender & \\
\hline Male & 35 \\
\hline Female & 12 \\
\hline Age: mean (SD), (range) & $60.6(11.5),(39-83)$ \\
\hline Mean BMI, $\mathrm{kg} / \mathrm{m}^{2}$ & 26.0 \\
\hline \multicolumn{2}{|l|}{ Cardiovascular risk factors, No. (\%) } \\
\hline Hypertension & $26(55)$ \\
\hline Diabetes & $18(38)$ \\
\hline Smoking & $21(48)$ \\
\hline Hyperlipidemia & $36(77)$ \\
\hline Mean heart rate, bpm: mean (SD), (range) & $69.2(12.2),(48-97)$ \\
\hline \multicolumn{2}{|l|}{ Heart rate ranged, No. (\%) } \\
\hline Ranged $48-69(\%)$ & $23(49)$ \\
\hline Ranged 70 - $97(\%)$ & $24(51)$ \\
\hline Agatston score: mean (SD), (range) & $507.7(590.5),(0-2328)$ \\
\hline \multicolumn{2}{|l|}{ Agatston score ranged, No. (\%) } \\
\hline Ranged $0-400(\%)$ & $25(57)$ \\
\hline Ranged 401- $2328(\%)$ & $19(43)$ \\
\hline
\end{tabular}

\subsection{Conventional Coronary Angiography}

In total, 189 stenoses ( $29.4 \%$ of all assessable segments) with a luminal narrowing of more than 50\% were found in 41 (87\%) patients using CCA. Significant coronary artery stenosis was excluded in six (13\%) of the patients, single vessel disease was present in six (13\%) patients, two-vessel disease in $11(23 \%)$ and three-vessel disease in 24 (51\%) of the patients.

In total, 642 segments out of potentially 705 segments (15 segments in each patient) were visualized during CCA and 103 segments could not be visualized due to the following reasons: proximal vessel occlusion, an anatomically absent vessel and a vessel diameter below $1.5 \mathrm{~mm}$. In addition, because of anatomic variations, 40 segments were added due to the following reasons: existence of ramus intermedius, PDA derived from LCX in left-dominant or co-dominant patients, and RCA branches with diameters more than $1.5 \mathrm{~mm}$.

\subsection{Dual-Source Computerized Tomography Coronary Angiog- raphy}

Out of 642 segments assessed in CCA, 628 segments (97.8\%) were visualized with diagnostic image quality in DSCT coronary angiography. Non-assessable image quality in $2.2 \%$ of segments was mainly due to motion artifact, vessel calcification, and low vessel opacification. Tables 2 and 3 show the diagnostic accuracy of DSCT coronary angiography compared to CCA results.

Table 2. Diagnostic Accuracy of DSCTA Compared with CCA in Detecting Stenosis More Than 50\% in Assessed Segments and Vessels

\begin{tabular}{lcc}
\hline & Segments $(\mathbf{n}=\mathbf{6 2 8})$ & Vessels $(\mathbf{n}=\mathbf{1 8 8})$ \\
\hline Sensitivity (95\% CI) & $93.7 \%(177 / 189)(88.9-$ & $97.1 \%(102 / 105)(91.3-$ \\
& $96.5)$ & $99.3)$ \\
\hline Specificity (95\% CI) & $96.8 \%(425 / 439(94.6-$ & $94.0 \%(78 / 83)(85.9-$ \\
& $98.2)$ & $97.8)$
\end{tabular}

Table 3. Segment-Based Diagnostic Accuracy of DSCTA for Detection of Stenosis More Than 50\% in Each Vessel Territory

\begin{tabular}{|c|c|c|c|}
\hline & LM-LAD & LCX & RCA \\
\hline $\begin{array}{l}\text { Sensitivity } \\
(95 \% \mathrm{CI})\end{array}$ & $\begin{array}{c}93.2 \%(82 / 88) \\
(85.2-97.2)\end{array}$ & $\begin{array}{c}88.2 \%(45 / 51) \\
(75.4-95.1)\end{array}$ & $\begin{array}{c}100 \%(50 / 50) \\
(91.1-100)\end{array}$ \\
\hline $\begin{array}{l}\text { Specificity } \\
\text { (95\% CI) }\end{array}$ & $\begin{array}{c}97.3 \%(143 / 147) \\
(92.7-99.1)\end{array}$ & $\begin{array}{c}95.6 \%(151 / 158) \\
(90.7-98.0)\end{array}$ & $\begin{array}{c}97.8 \%(131 / 134) \\
(93.1-99.4)\end{array}$ \\
\hline $\operatorname{PPV}(95 \%$ CI $)$ & $\begin{array}{c}95.3 \%(82 / 86) \\
(87.9-98.5)\end{array}$ & $\begin{array}{c}86.5 \%(45 / 52) \\
(73.6-94.0)\end{array}$ & $\begin{array}{c}94.3 \%(50 / 53) \\
(83.4-98.5)\end{array}$ \\
\hline NPV (95\% CI) & $\begin{array}{c}96.0 \%(143 / 149) \\
(91.1-98.4)\end{array}$ & $\begin{array}{c}96.2 \%(151 / 157) \\
(91.5-98.4)\end{array}$ & $\begin{array}{c}100 \%(131 / 131) \\
(96.4-100)\end{array}$ \\
\hline Accuracy & $95.7 \%$ & $93.8 \%$ & $98.4 \%$ \\
\hline
\end{tabular}

Abbreviation: LM, left main; LAD, left anterior descending; LCX, left circumflex; RCA, right coronary artery; CI, confidence interval; PPV, positive predictive value; NPV, negative predictive value

\subsection{Assessment Per Segment}

On per segment analysis, DSCT coronary angiography in comparison to CCA showed a sensitivity of $93.7 \%$ (177/189), specificity of $96.8 \%$ (425/439), PPV of $92.7 \%$ (177/191), and NPV of 97.2\% (425/437). PLR value was 29.4, while NLR was 0.066 (Table 2).

In total, DSCT coronary angiography produced 14 false positive and 12 false negative results. Due to the presence of multiple coronary ectasia in one patient, normal coronary segments were diagnosed as stenotic lesions, giving rise to three false positive results. Examples of three patients who 
underwent DSCT coronary angiography for evaluation of CAD are provided in Figures 1 and 2.
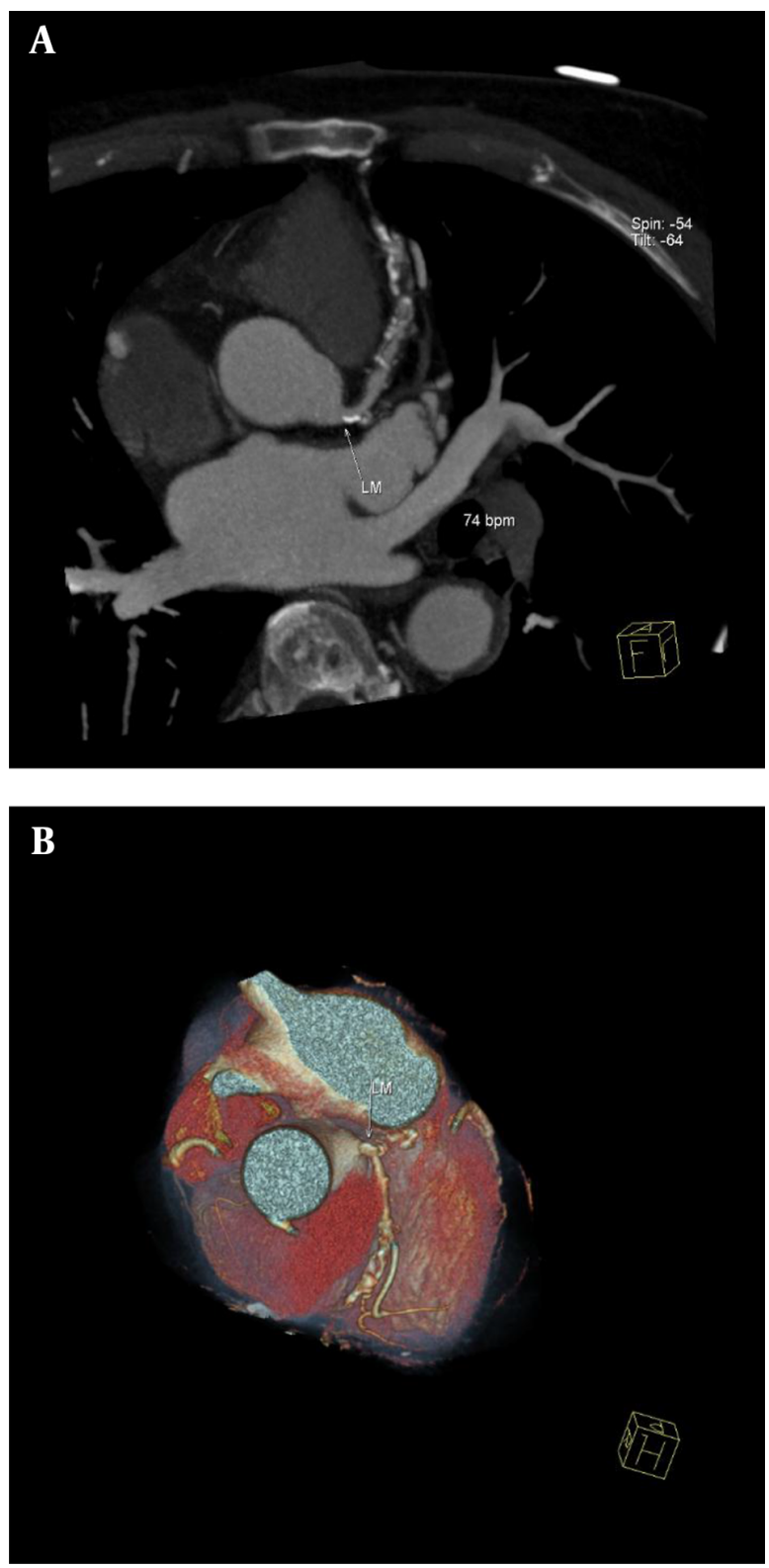

Figure 1. Dual-source CT coronary angiography of a 71-year-old man (mean heart rate during scanning, 74 beats per minute; Agatston score, 1801). A, A maximumintensity projection through the centerline of the left main coronary artery (LM) and left descending artery (LAD) demonstrates significant stenosis in LM and LAD; B Conventional coronary angiography showed no significant stenosis in LM, but significant stenosis was seen in LAD. False positive result was the consequence of a calcified plaque in LM. Volume rendered image of the same patient showed severe tor tuosity in LAD.
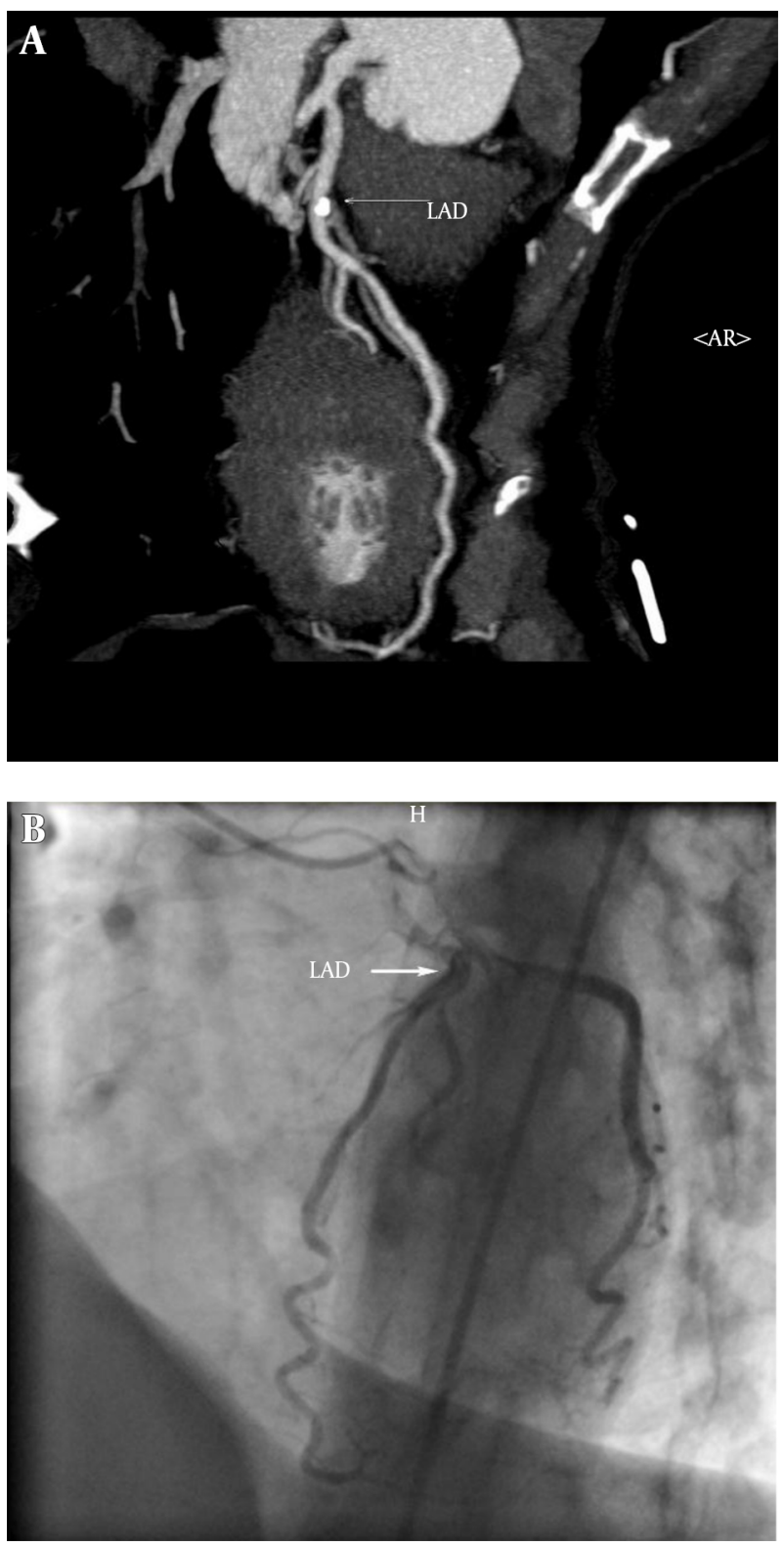

Figure 2. Dual-source CT coronary angiography of a 51-year-old woman (mean heart rate during scanning, 81 beats per minute; Agatston score, 94). A thin-slab maximum-intensity projection of left descending artery (LAD) demonstrates two non-significant coronary stenosis in proximal and mid segments. A, The first stenosis has a calcified plaque and the second one has a non-calcified plaque; B, conventional coronary angiography reveals that both of the stenoses were non-significant, hence dual-source CT coronary angiography correctly showed stenoses as nonsignificant.

\subsection{Assessment Per Vessel}

Comparison of DSCT coronary angiography to CCA on per vessel basis (presence of at least one significant stenosis or absence of any significant stenosis in each vessel) produced following diagnostic accuracy parameters for DSCT 
coronary angiography: Sensitivity, 97.1\% (102/105); specificity, 94.0\% (78/83); PPV, 95.3\% (102/107); NPV, 96.3\% (78/81); PLR, 16.1; and NLR, 0.030 (Table 2).

\subsection{Diagnostic Assessment in Segments Pertaining to Various Coronary Vessel Territories}

This analysis was performed to assess the diagnostic performance of DSCT coronary angiography in the territory of each main coronary artery. RCA territory included segments $1-4$, and other RCA branches with diameters above $1.5 \mathrm{~mm}$; LCX territory included segments 11 - 15, segment 16 and PDA originating from LCX, if present; LMCA and LAD (LM-LAD) territory included segments 5 - 10. Diagnostic accuracy of DSCT coronary angiography in separate territories is summarized in Table 3.

\subsection{Non-Coronary Findings in DSCT Coronary Angiography}

In addition to evaluating coronary arteries, DSCT coronary angiography produced some non-coronary findings as well. A mass lesion in the right lung was found in one patient who was then referred for further evaluation. Pericardial effusion in one patient and apical clots in three patients were also unexpectedly found analyzing the CTCA images.

\section{Discussion}

The results of the present study showed that DSCT coronary angiography has clinically acceptable accuracy in the diagnosis of coronary artery stenosis among patients who were referred to our center during daily clinical practice.

Likelihood ratio (LR) is a useful index of any diagnostic test. Unlike sensitivity and specificity, LR can be used at the individual patient level and it individualizes the probability of a diagnosis. Furthermore, these measures are based on a ratio of sensitivity and specificity, hence not varying in different populations or settings (22). For a given diagnostic test, LRs greater than 1 indicate that the test result is associated with the disease, and values closer to zero indicate that the finding decreases the probability of the disease. Generally, LRs above 10 and below 0.1 are considered to provide strong evidence to rule in or rule out diagnosis $(22,23)$. Good LR values of current study confirmed that DSCT coronary angiography provides a high diagnostic accuracy for evaluation of CAD in daily clinical practice.

Our findings are consistent with several other studies that have previously investigated the diagnostic performance of DSCT coronary angiography. In a study by Scheffel and colleagues, per segment analysis of DSCT coronary angiography findings showed a sensitivity of $96.5 \%$ and specificity of 97.5\%. Also PPV and NPV of DSCT coronary angiography were reported as $85.7 \%$ and $99.4 \%$, respectively (10). The results of our study showed a lower accuracy for DSCT coronary angiography compared to the study conducted by Scheffel et al. This could be explained by the difference in the setting of the study and recruitment of the subjects. While in the study by Scheffel et al., all the subjects were recruited with specific inclusion and exclusion criteria, we selected our subjects from patients who were referred to our center during daily clinical practice and aimed to show the accuracy of DSCT coronary angiography while it is used in a real clinical setting and not a research setting. Cademartiri et al. also tested the accuracy of CTCA in the real world setting and they have reached a similar conclusion (24).

In a recent meta-analysis (25) on the diagnostic performance of DSCT coronary angiography, the pooled diagnostic parameters of per segment analysis were reported as follows: sensitivity, 95\%; specificity, 97\%; PLR, 35.2; and NLR , 0.05, which are in accordance with the current study. In addition, per vessel analysis of the DSCT findings showed a sensitivity, specificity, PLR, and NLR of 97\%, 94\%, 16.4, and 0.03 , respectively which are in line with our findings.

In total, 14 segments (2.2\%) were non-assessable in our study. This value is comparable to the systematic review by Salavati et al. (14) that evaluated 21 studies and reported that $2 \%$ of the segments which were studied by CTCA were non-assessable.

The current study was performed in a daily routine clinical setting, which makes it different in some aspects compared to other studies. In routine practice, a high number of diagnostic studies should be performed in the radiology department every day. This limits the evaluation time that is spent for each DSCT coronary angiography study and might lead to the decrease of the diagnostic accuracy of DSCT compared to the studies that performed DSCT coronary angiography in a research setting. Also, considering the time limitation in a daily routine clinical setting, all the evaluations were done visually and this could also be considered as another cause of discrepancy between DSCT and CCA.

In a systematic review by Mowatt G. et al. it was found that the diagnostic accuracy of CTCA for the evaluation of stenosis of LCX artery is lower than other major coronary arteries (15). It was hypothesized that this might be due to the anatomical position of the LCX artery, which is close to the atrium, and thereby is more affected by atrial contraction (26). Similarly, in our study, it was found that the diagnostic accuracy of LCX is slightly lower than LM-LAD and RCA, although the difference was not significant. The fact that no significant difference in the diagnostic accuracy of DSCT findings between RCA, LM-LAD, and LCX artery terri- 
tories were found, might imply that the diagnostic performance of DSCT is not much influenced by temporal resolution (27).

Conventional coronary angiography still remains the gold standard for diagnosing coronary aneurysm and ectasia. However, CCA provides no information about the vessel wall (28). Therefore, CCA may underestimate the size of the aneurysm, and when it is occluded or contains thrombosis, the aneurysm may not even be seen on CCA (29). Excellent correlation between MDCT coronary angiography and CCA has been reported in the detection of coronary aneurysm and stenoses in patients after childhood Kawasaki syndrome (30). However in our study, normal segments of the artery between multiple aneurysms were reported as significant stenosis, in one patient, ensuing in false positive results for DSCT imaging study. Therefore, interpretation of luminal stenosis in patients with coronary aneurysms needs special consideration.

The capability of depicting additional findings is one of the unique features of the CT angiography technique. According to the American college of radiology guidelines, interpretation of non-cardiac findings are necessary in CTCA imaging studies (31). Lazoura et al. demonstrated incidental non-cardiac findings in as high as $56 \%$ of the patients undergoing CTCA (32). Similar to our study, others have reported findings such as pleural effusion, pulmonary embolus, and pulmonary malignancy $(33,34)$. This feature might even help to determine the cause of chest pain or respiratory symptoms in patients with normal coronary arteries (35).

The retrospective nature of the study confined our control over the use of daily beta-blockers by the subjects, although an additional beta-blocker was not prescribed for heart rate control before CTCA study. While this is a retrospective study, we did not ask patients to do both of the examinations. As a substitute, we chose patients who underwent both DSCT coronary angiography and CCA in a 60day period. So, this could lead to a high prevalence of CAD in our patient population. PPV and NPV are influenced by disease prevalence; consequently, the ability of DSCT to detect and to rule out stenosis could be overestimated. However, this limitation could be balanced with the use of LR in our study. Moreover, we should state that current results are reached in a population with a high prevalence of CAD, which is the favorable population presented to a referral cardiovascular center. The small number of patients was another limitation of the present study.

In conclusion, our results indicate that DSCT coronary angiography provides a high diagnostic accuracy for evaluation of CAD in the daily routine practice of a referral cardiovascular setting. These results are comparable to studies performed in a research setting. Moreover, acceptable
LR values of the current study demonstrate that secondgeneration DSCT coronary angiography is a reliable tool that could be used as a non-invasive method for assessment of CAD in the clinical setting.

\section{Acknowledgments}

We thank Rajaei imaging center personnel for their precious help particularly Ms. Hekmat and Ms. Garakany.

\section{Footnotes}

Authors' Contribution: Study design was carried out by Marzieh Motevalli, Ali Mohammadzadeh, and Arash Mahdavi; data acquisition was performed by Arash Mahdavi, and Mohammad Reza Tabatabaei; data interpretation was accomplished by Marzieh Motevalli, Ali Mohammadzadeh, and Farhad Sheikholeslami; data analysis was done by Arash Mahdavi, Golsa Joodi, Farhad Sheikholeslami, and Mohammad Reza Tabatabaei; literature research was achieved by Arash Mahdavi, Golsa Joodi, and Ali Mohammadzadeh; Manuscript drafting and manuscript editing were completed by Arash Mahdavi, Golsa Joodi, and Marzieh Motevalli.

Conflict of Interest: None declared.

Financial Disclosure: This study was not funded by any institution.

Funding/Support: This study was not funded by any institution.

\section{References}

1. Go AS, Mozaffarian D, Roger VL, Benjamin EJ, Berry JD, Borden WB, et al. Heart disease and stroke statistics-2013 update: a report from the American Heart Association. Circulation. 2013;127(1):e6-e245. doi: 10.1161/CIR.ob013e31828124ad. [PubMed: 23239837].

2. Azizi F, Salehi P, Etemadi A, Zahedi-Asl S. Prevalence of metabolic syndrome in an urban population: Tehran Lipid and Glucose Study. DIABETES RES CLIN PR. 2003;61(1):29-37. doi:10.1016/s0168-8227(03)000664.

3. Bax JJ, Schuijf JD. Which role for multislice computed tomography in clinical cardiology?. Am Heart J. 2005;149(6):960-1. doi: 10.1016/j.ahj.2005.03.048. [PubMed: 15976774].

4. Yang X, Gai LY, Li P, Chen YD, Li T, Yang L. Diagnostic accuracy of dual-source CT angiography and coronary risk stratification. Vasc Health Risk Manag. 2010;6:935-41. doi:10.2147/VHRM.S13879. [PubMed: 21057578].

5. Patel MR, Peterson ED, Dai D, Brennan JM, Redberg RF, Anderson HV, et al. Low diagnostic yield of elective coronary angiography. $N$ Engl J Med. 2010;362(10):886-95. doi: 10.1056/NEJMoa0907272. [PubMed: 20220183].

6. Schuetz GM, Zacharopoulou NM, Schlattmann P, Dewey M. Metaanalysis: noninvasive coronary angiography using computed tomography versus magnetic resonance imaging. Ann Intern Med. 2010;152(3):167-77. doi: 10.7326/0003-4819-152-3-201002020-00008. [PubMed: 20124233]. 
7. Leber AW, Johnson T, Becker A, von Ziegler F, Tittus J, Nikolaou K, et al. Diagnostic accuracy of dual-source multi-slice CT-coronary angiography in patients with an intermediate pretest likelihood for coronary artery disease. Eur Heart J. 2007;28(19):2354-60. doi: 10.1093/eurheartj/ehm294. [PubMed: 17644815].

8. Raff GL, Gallagher MJ, O’Neill WW, Goldstein JA. Diagnostic accuracy of noninvasive coronary angiography using 64-slice spiral computed tomography. J Am Coll Cardiol. 2005;46(3):552-7. doi: 10.1016/j.jacc.2005.05.056. [PubMed: 16053973].

9. Mollet NR, Cademartiri F, van Mieghem CA, Runza G, McFadden EP, Baks T, et al. High-resolution spiral computed tomography coronary angiography in patients referred for diagnostic conventional coronary angiography. Circulation. 2005;112(15):2318-23. doi: 10.1161/CIRCULATIONAHA.105.533471. [PubMed: 16203914].

10. Scheffel H, Alkadhi H, Plass A, Vachenauer R, Desbiolles L, Gaemperli O, et al. Accuracy of dual-source CT coronary angiography: First experience in a high pre-test probability population without heart rate control. Eur Radiol. 2006;16(12):2739-47. doi: 10.1007/s00330-006-0474-0. [PubMed: 17031451].

11. Abdulla J, Abildstrom SZ, Gotzsche O, Christensen E, Kober L, TorpPedersen C. 64-multislice detector computed tomography coronary angiography as potential alternative to conventional coronary angiography: a systematic review and meta-analysis. Eur Heart J. 2007;28(24):3042-50. doi: 10.1093/eurheartj/ehm466. [PubMed: 17981829].

12. Shabestari AA, Abdi S, Akhlaghpoor S, Azadi M, Baharjoo H, Pajouh $\mathrm{MD}$, et al. Diagnostic performance of 64-channel multislice computed tomography in assessment of significant coronary artery disease in symptomatic subjects. Am J Cardiol. 2007;99(12):1656-61. doi: 10.1016/j.amjcard.2007.01.040. [PubMed: 17560870].

13. Nazeri I, Shahabi P, Tehrai M, Sharif-Kashani B, Nazeri A. Impact of calcification on diagnostic accuracy of 64-slice spiral computed tomography for detecting coronary artery disease: a single center experience. Arch Iran Med. 2010;13(5):373-83. doi: 010135/AIM.003. [PubMed: 20804303].

14. Salavati A, Radmanesh F, Heidari K, Dwamena BA, Kelly AM, Cronin P. Dual-source computed tomography angiography for diagnosis and assessment of coronary artery disease: systematic review and meta-analysis. J Cardiovasc Comput Tomogr. 2012;6(2):78-90. doi: 10.1016/j.jcct.2011.10.018. [PubMed: 22226727].

15. Mowatt G, Cummins E, Waugh N, Walker S, Cook JA, Jia X, et al. Systematic review of the clinical effectiveness and cost-effectiveness of 64-slice or higher computed tomography angiography as an alternative to invasive coronary angiography in the investigation of coronary artery disease. Health Technol Assess. 2008;12(17).

16. Moon JH, Park EA, Lee W, Yin YH, Chung JW, Park JH, et al. The diagnostic accuracy, image quality and radiation dose of 64-slice dual-source CT in daily practice: a single institution's experience. Korean J Radiol. 2011;12(3):308-18. doi: 10.3348/kjr.2011.12.3.308. [PubMed: 21603290].

17. Flohr TG, McCollough CH, Bruder H, Petersilka M, Gruber K, Suss C, et al. First performance evaluation of a dual-source CT (DSCT) system. Eur Radiol. 2006;16(2):256-68. doi: 10.1007/s00330-005-2919-2. [PubMed: 16341833].

18. Cademartiri F, Mollet N, van der Lugt A, Nieman K, Pattynama PM, de Feyter PJ, et al. Non-invasive 16-row multislice CT coronary angiography: usefulness of saline chaser. Eur Radiol. 2004;14(2):178-83. doi: 10.1007/s00330-003-2188-x. [PubMed: 14689227].

19. Agatston AS, Janowitz WR, Hildner FJ, Zusmer NR, Viamonte M, Detrano R. Quantification of coronary artery calcium using ultrafast computed tomography. J Am Coll Cardiol. 1990;15(4):827-32. doi: 10.1016/0735-1097(90)90282-t.

20. Austen WG, Edwards JE, Frye RL, Gensini GG, Gott VL, Griffith LS, et al A reporting system on patients evaluated for coronary artery disease. Report of the Ad Hoc Committee for Grading of Coronary Artery Dis- ease, Council on Cardiovascular Surgery, American Heart Association. Circulation. 1975;51(4 Suppl):5-40. [PubMed: 1116248].

21. Gouya H, Varenne O, Trinquart L, Touze E, Vignaux O, Spaulding C, et al Coronary artery stenosis in high-risk patients: 64-section CT and coronary angiography-prospective study and analysis of discordance. $R a$ diology. 2009;252(2):377-85. doi: 10.1148/radiol.2522081271. [PubMed: 19546426].

22. Deeks JJ, Altman DG. Diagnostic tests 4: likelihood ratios. BMJ. 2004;329(7458):168-9. doi: 10.1136/bmj.329.7458.168. [PubMed: 15258077].

23. McGee S. Simplifying likelihood ratios. $J$ Gen Intern Med. 2002;17(8):646-9. [PubMed: 12213147].

24. Cademartiri F, Maffei E, Notarangelo F, Ugo F, Palumbo A, Lina D, et al. 64-slice computed tomography coronary angiography: diagnostic accuracy in the real world. Radiol Med. 2008;113(2):163-80. doi: 10.1007/s11547-008-0241-1. [PubMed: 18386119].

25. Li M, Zhang GM, Zhao JS, Jiang ZW, Peng ZH, Jin ZT, et al. Diagnostic performance of dual-source CT coronary angiography with and without heart rate control: systematic review and meta-analysis. Clin Radiol. 2014;69(2):163-71. doi:10.1016/j.crad.2013.09.008. [PubMed: 24268513]

26. Pannu HK, Flohr TG, Corl FM, Fishman EK. Current concepts in multidetector row CT evaluation of the coronary arteries: principles, techniques, and anatomy. Radiographics. 2003;23 Spec No:S111-25. doi: 10.1148/rg.23si035514. [PubMed: 14557506].

27. Herzog C, Zwerner PL, Doll JR, Nielsen CD, Nguyen SA, Savino G, et al. Significant coronary artery stenosis: comparison on per-patient and per-vessel or per-segment basis at 64-section CT angiography. $R a$ diology. 2007;244(1):112-20. doi: 10.1148/radiol.2441060332. [PubMed: 17581898].

28. Diaz-Zamudio M, Bacilio-Perez U, Herrera-Zarza MC, Meave-Gonzalez A, Alexanderson-Rosas E, Zambrana-Balta GF, et al. Coronary artery aneurysms and ectasia: role of coronary CT angiography. Radiographics. 2009;29(7):1939-54. doi: 10.1148/rg.297095048. [PubMed: 19926755].

29. LaMotte LC, Mathur VS. Atherosclerotic coronary artery aneurysms: eight-year angiographic follow-up. Tex Heart Inst J. 2000;27(1):72-3. [PubMed: 10830637].

30. Arnold R, Ley S, Ley-Zaporozhan J, Eichhorn J, Schenk JP, Ulmer H, et al. Visualization of coronary arteries in patients after childhood Kawasaki syndrome: value of multidetector CT and MR imaging in comparison to conventional coronary catheterization. Pediatr Radiol. 2007;37(10):998-1006. doi:10.1007/s00247-007-0566-2. [PubMed: 17768616].

31. Jacobs JE, Boxt LM, Desjardins B, Fishman EK, Larson PA, Schoepf J, et al. ACR practice guideline for the performance and interpretation of cardiac computed tomography (CT). J Am Coll Radiol. 2006;3(9):67785. doi: 10.1016/j.jacr.2006.06.006. [PubMed: 17412148].

32. Lazoura O, Vassiou K, Kanavou T, Vlychou M, Arvanitis DL, Fezoulidis IV. Incidental non-cardiac findings of a coronary angiography with a 128-slice multi-detector CT scanner: should we only concentrate on the heart?.Korean J Radiol. 2010;11(1):60-8. doi: 10.3348/kjr.2010.11.1.60. [PubMed: 20046496].

33. Dewey M, Schnapauff D, Teige F, Hamm B. Non-cardiac findings on coronary computed tomography and magnetic resonance imaging. Eur Radiol. 2007;17(8):2038-43. doi: 10.1007/s00330-006-0570-1. [PubMed: 17268800].

34. Onuma Y, Tanabe K, Nakazawa G, Aoki J, Nakajima H, Ibukuro K, et al. Noncardiac findings in cardiac imaging with multidetector computed tomography. J Am Coll Cardiol. 2006;48(2):402-6. doi: 10.1016/j.jacc.2006.04.071. [PubMed: 16843193].

35. Halpern EJ. Triple-rule-out CT angiography for evaluation of acute chest pain and possible acute coronary syndrome. Radiology. 2009;252(2):332-45. doi: 10.1148/radiol.2522082335. [PubMed: 19703877]. 